\title{
A CONSCIÊNCIA FONOLÓGICA E A CONSCIÊNCIA DO PRÓPRIO DESVIO DE FALA NAS DIFERENTES GRAVIDADES DO DESVIO FONOLÓGICO
}

\section{Phonological awareness and awareness of the own speech impairment in different severity levels of phonological disorder}

\author{
Roberta Freitas Dias ${ }^{(1)}$, Helena Bolli Mota ${ }^{(2)}$, Carolina Lisbôa Mezzomo ${ }^{(3)}$
}

\begin{abstract}
RESUMO
Objetivo: verificar a relação das habilidades em consciência fonológica e da consciência do próprio desvio de fala com diferentes gravidades do desvio fonológico. Métodos: 14 crianças participaram desse estudo, com idades entre 5:0 e 6:11;29, apresentando diagnóstico de desvio fonológico. Os desvios fonológicos foram classificados de duas formas: quantitativa e qualitativa. Conforme a classificação quantitativa foram formados os grupos Desvio Médio, Desvio Médio-Moderado e Desvio Moderado-Severo. Seguindo a classificação qualitativa, foram formados o grupo com características Atrasadas e o grupo com características Iniciais. Para a obtenção dos dados foram aplicados o Protocolo de Tarefas de Consciência Fonológica e o Teste de consciência do próprio desvio de fala. Os dados foram analisados por meio do teste estatístico Kruskal-Wallis. Resultados: diferenças estatisticamente significantes foram observadas no desempenho das tarefas de realismo nominal e de detecção de sílaba entre os grupos classificados de maneira qualitativa. Para os grupos classificados de forma quantitativa não houve diferença estatisticamente significante em nenhuma das tarefas de consciência fonológica. Contudo, quanto à consciência do próprio desvio de fala, somente estes últimos grupos apresentaram diferença estatisticamente significante entre si. Conclusão: identificou-se uma possível relação entre o desempenho nas tarefas de consciência fonológica e as diferentes gravidades dos desvios fonológicos, principalmente quando classificados de forma qualitativa. A consciência do próprio desvio de fala também parece sofrer influência das diferentes gravidades do desvio fonológico, sobretudo, quando classificados de forma quantitativa.
\end{abstract}

DESCRITORES: Fala; Distúrbios da Fala; Criança; Linguagem

(1) Fonoaudióloga; Bolsista Coordenação de Aperfeiçoamento de Pessoal de Nível Superior, CAPES; Mestranda em Distúrbios da Comunicação Humana pela Universidade Federal de Santa Maria.

(2) Fonoaudióloga; Professora Associada do Curso de Fonoaudiologia da Universidade Federal de Santa Maria e do Curso de Pós-Graduação em Distúrbios da Comunicação Humana da Universidade Federal de Santa Maria, UFSM, Santa Maria, RS; Mestre e Doutora em Linguística Aplicada pela Pontifícia Universidade Católica do Rio Grande do Sul.

(3) Fonoaudióloga; Professora Adjunto II do Curso de Fonoaudiologia da Universidade Federal de Santa Maria e do Curso de Pós-Graduação em Distúrbios da Comunicação Humana da Universidade Federal de Santa Maria, UFSM, Santa Maria RS; Especialista em Linguagem; Especialista em Motricidade Oral; Mestre e Doutora em Letras pela Pontifícia Universidade Católica do Rio Grande do Sul.

Conflito de interesse: inexistente

\section{INTRODUÇÃO}

A aquisição fonológica normal ocorre quando, dentro de uma determinada faixa etária (aproximadamente dos quatro aos seis anos de idade), o domínio do sistema fonológico da língua-alvo é atingido espontaneamente em uma sequência comum à maior parte das crianças ${ }^{1-3}$. Por outro lado, o desenvolvimento fonológico desviante não ocorre da mesma forma, pois a construção do conhecimento linguístico resulta em um sistema fonológico distinto do input recebido, do alvo-adulto e, portanto, inadequado em relação a este. Esse grupo de indivíduos pode apresentar um inventário fonético incompleto em relação ao modelo da sua comunidade linguística ${ }^{2,4,5}$. 
O desvio fonológico (DF) pode ser classificado em diferentes gravidades por meio de uma análise quantitativa, como o cálculo da gravidade do desvio pelo Percentual de Consoantes Corretas (PCC) ${ }^{6,7}$ ou, por uma análise qualitativa que propõe uma tipologia para os $\mathrm{DF}^{3,8}$.

A classificação denominada Percentage of Consonants Corrects - PCC se faz por meio de uma análise quantitativa. Nessa classificação verifica-se o número de consoantes corretas produzidas em um número total de consoantes obtidas em uma amostra de fala espontânea. O PCC é obtido com a divisão do número de consoantes corretas pelo número de consoantes corretas somado ao número de consoantes incorretas, multiplicado por 100. Com isso, tem-se a seguinte classificação: Desvio Médio (PCC de 85 a 100\%); Desvio Médio-Moderado (PCC de 65 a 85\%); Desvio Moderado-Severo (PCC de 50 a 65\%) e Desvio Severo (PCC igual ou inferior a $50 \%)^{6}$.

A classificação qualitativa propõe uma tipologia para os DF e é estabelecida de acordo com características apresentadas no sistema fonológico considerando os processos fonológicos. Na classificação utilizada neste trabalho são identificados quatro grupos com características diferentes ${ }^{8}$ :

- Desvio Fonológico com características Incomuns - Incomuns;

- Desvio Fonológico com características Iniciais - Iniciais;

- Desvio Fonológico com características Atrasadas - Atrasadas;

- Desvio Fonológico com características Fonéticas Adicionais - Fonéticas.

Há crianças com DF que parecem ter acesso a representações fonológicas normais, pois têm consciência da sua fonologia desviante. A consciência da falha leva as crianças a fazerem tentativas de transmitir o sentido correto das palavras, o que não significa necessariamente que elas tenham considerado a estrutura linguística ou realizado manipulações conscientes das formas linguísticas para transmitir o seu significado. Somente quando se tem uma clara evidência de que as crianças desprezam o conteúdo e refletem a respeito fazendo comentários explícitos sobre, ou manipulando conscientemente formas e características linguísticas, é que se pode afirmar que são metalinguisticamente conscientes ${ }^{2,9}$.

Crianças com DF podem ter consciência do sistema fonológico considerado normal, uma vez que, são capazes de refletir sobre os sons da fonologia de um sistema por meio das habilidades em Consciência Fonológica (CF). Elas podem reconhecer os desvios de fala cometidos por elas mesmas, ou seja, podem ter consciência do próprio desvio ${ }^{10}$.
CF é uma habilidade cognitiva que permite a reflexão sobre os sons da fala. Essa habilidade pressupõe a capacidade de identificar que as palavras são constituídas por sons, os quais podem ser manipulados conscientemente. Isto significa dizer que a CF permite à criança reconhecer que as palavras rimam, terminam ou começam com o mesmo som e são compostas por sons individuais que podem ser manipulados para a formação de novas palavras ${ }^{11-14}$.

As crianças com DF têm, de forma geral, um nível de consciência metalinguística inferior quando comparadas a crianças com desenvolvimento fonológico normal. O tipo de conhecimento linguístico que as crianças com DF têm acesso pode justificar algumas dificuldades encontradas por elas para desempenhar tarefas metalinguísticas. Ainda que essas crianças tenham acesso a um tipo de conhecimento fonológico e capacidade para análise como têm as crianças normais, suas respostas às tarefas linguísticas serão incorretas, já que sua representação fonológica é diferente ${ }^{2,9,15,16}$.

No Brasil, as pesquisas envolvendo habilidades em CF são desenvolvidas principalmente com crianças com desenvolvimento fonológico normal, buscando traçar um perfil de CF infantil e, também, no que se refere à alfabetização. Com isso, é interessante e necessário que estudos em CF envolvendo DF de diferentes gravidades sejam desenvolvidos. O conhecimento destas habilidades neste grupo de crianças pode contribuir não só para a terapia fonoaudiológica, mas também, para o processo de alfabetização futuro.

Este estudo teve como objetivo verificar a relação do desempenho em habilidades metalinguísticas, CF e Consciência do Próprio Desvio de Fala (CPDF), com as diferentes gravidades do DF considerando diferentes classificações - quantitativa e qualitativa.

\section{MÉTODOS}

Foi realizada uma pesquisa exploratória de corte transversal em que participaram 14 crianças, oito meninos e seis meninas, na faixa etária de 5:0 a 6:11, com diagnóstico de DF. Essas crianças foram selecionadas a partir das triagens do setor de fala do Serviço de Atendimento Fonoaudiológico da Universidade Federal de Santa Maria - Santa Maria/ RS e de uma escola estadual desta mesma cidade. A escola foi escolhida a partir de uma pesquisa de onde provém o maior número de pacientes atendidos no setor de fala do Serviço de Atendimento Fonoaudiológico para que dessa forma, fosse homogeneizado o nível sócio-econômico-cultural dos participantes desse estudo. 
Para que as crianças fossem incluídas na amostra foi necessário que atendessem aos seguintes critérios: ter idade entre 5:00 e 6:11;29; apresentar nível de escrita pré-silábico ou silábico; apresentar diagnóstico de DF e não ter realizado e/ou iniciado terapia fonoaudiológica.

A partir das análises das avaliações fonoaudiológicas realizadas no Serviço de Atendimento Fonoaudiológico foi possível selecionar os indivíduos que apresentavam DF nesta instituição. Já na escola, foi realizada triagem fonoaudiológica em que as crianças passaram por avaliação dos órgãos fonoarticulatórios, avaliação da linguagem, avaliação da fala, além da triagem auditiva. A fim de padronizar o grupo, foi realizada a avaliação do nível da escrita sendo necessário que as crianças apresentassem os níveis pré-silábico ou silábico já que se tem conhecimento de que determinadas habilidades em CF podem se desenvolver com a alfabetização.

A avaliação do sistema estomatognático foi realizada de acordo com o protocolo de avaliação disponível no Serviço de Atendimento Fonoaudiológico da Universidade Federal de Santa Maria, no qual foram observados aspecto, postura, tônus e mobilidade dos órgãos fonoarticulatórios e suas funções - respiração, sucção, mastigação e deglutição. A avaliação da linguagem e a avaliação da fala, foram realizadas por meio de uma sequência lógica de quatro fatos (ilustração de um aniversário). As figuras foram apresentadas à criança fora da ordem, sendo incentivado que ela as organizasse e contasse uma história espontâneamente. Observou-se com isso, aspectos da linguagem expressiva oral, tais como sintaxe, morfologia, semântica, pragmática, verificando se estavam coerentes e adequados a sua idade. Foram observados também a ocorrência de alterações na produção dos fonemas. A linguagem compreensiva foi observada mediante perguntas que o avaliador foi fazendo durante e após o conto da história no sentido, também, de incentivar a narrativa ${ }^{17}$.

A triagem auditiva foi realizada no Serviço de Atendimento Fonoaudiológico e nas escolas, com a inspeção do meato acústico externo e pesquisa dos limiares auditivos tonais. As crianças que apresentaram alterações nas avaliações de audição, dos órgãos fonoarticulatórios e linguagem foram excluídas da amostra. O diagnóstico de DF foi realizado pelas autoras, após a análise de todas as avaliações realizadas.

Para a avaliação do sistema fonético/fonológico foi aplicado a Avaliação Fonológica da Criança (AFC) ${ }^{4}$. Esse instrumento é constituído de cinco desenhos temáticos (banheiro, cozinha, sala, veículos, e zoológico) que possibilitam a obtenção de uma amostra de fala, por meio de nomeação e fala espontânea, contendo todos os fones contrastivos do Português em todas as posições que podem ocorrer em relação à estrutura da sílaba e da palavra. As gravações de fala obtidas para o AFC foram transcritas e revisadas por pelo menos um julgador com experiência em transcrição fonética - bolsistas de iniciação científica do Centro de Estudos de Linguagem e Fala/Curso de Fonoaudiologia/UFSM separadamente. Com a avaliação do sistema fonético/fonológico os DF foram classificados de forma quantitativa (Desvio Médio; Desvio Médio-Moderado; Desvio Moderado-Severo e Desvio Severo) ${ }^{6}$ e qualitativa (Incomuns; Iniciais; Atrasadas e Fonéticas) ${ }^{8}$.

A classificação quantitativa - PCC - é obtida por meio do percentual de consoantes corretas produzidas em um número total de consoantes obtidas em uma amostra de fala espontânea. O valor obtido com a divisão do número de consoantes corretas pelo número de consoantes corretas somado ao número de consoantes incorretas, multiplicado por 100 revela o grau de gravidade do $\mathrm{DF}^{6}$.

Quanto à classificação qualitativa, consideram-se as características identificadas no sistema fonológico da criança. Nos DF com características Incomuns são observados processos fonológicos incomuns como, por exemplo, fricatização, glotalização e apagamento de fricativa/plosiva, sendo o sistema fonológico bastante defasado em relação ao sistema padrão de crianças mais jovens com desenvolvimento fonológico normal. Os inventários fonético e fonológico são restritos, podendo haver preferência sistemática por um som. Em geral, somente pessoas de convívio diário identificam o que está sendo dito por essas crianças. Nas Iniciais observam-se características específicas do desenvolvimento inicial da aquisição da linguagem com alguns processos que já deveriam ter sido suprimidos, como por exemplo, plosivização, anteriorização de plosiva, dessonorização. Há ininteligibilidade de fala, no entanto, em um grau menos severo. Nos DF com características Atrasadas tem-se um sistema com "simples atraso" em relação à etapa de aquisição. São alterações evidenciadas no estágio final da aquisição fonológica normal apresentando um sistema com alterações envolvendo, em geral, as fricativas palatais e as líquidas, e noções de estrutura silábica (CVC e CCV), o que determina processos comuns da fala infantil e uma fala um pouco ininteligível. Nas fonéticas, fatores como, por exemplo, freio lingual curto, otites frequentes e respiração oral, que interferem no desenvolvimento e adequação do sistema fonológico são observados. Pode-se verificar que tais características 
enquadram-se plenamente nas características dos outros grupos descritos acima ${ }^{8}$.

A avaliação da escrita baseou-se na classificação dos níveis de escrita em pré-silábico, silábico, silábico-alfabético e alfabético ${ }^{18}$.

A avaliação das habilidades em CF foi realizada por meio do Protocolo de Tarefas de Consciência Fonológica (PTCF) ${ }^{13}$, do qual foram aplicadas apenas as tarefas que o teste propõe para a faixa etária dos cinco aos seis anos de idade, de acordo com as instruções do teste. As seguintes tarefas foram aplicadas:

- Segmentação de frases em palavras (T1) utilizando-se frases de duas, três e quatro palavras;

- Realismo Nominal (T2);

- Detecção de rimas (T3) - utilizando-se palavras dissílabas e trissílabas;

- Síntese silábica (T4) - utilizando-se palavras dissílabas, trissílabas e quadrissílabas;

- Segmentação silábica (T5) - utilizando-se palavras dissílabas, trissílabas e quadrissílabas;

- Detecção de sílabas (T6) - utilizando-se palavras com sílabas iguais em posição inicial, medial e final;

- Reversão silábica (T7) - utilizando-se apenas palavras dissílabas;

- Detecção de fonemas (T9) - utilizando-se palavras com fonemas iguais na posição inicial e final.

- Seguindo as instruções do teste, para as crianças na faixa etária dos 5:0 foram aplicadas todas as tarefas do PTCF descritas, exceto as subtarefas T7 e T9 final. Já para as crianças na faixa etária dos 6:0, não foram aplicadas somente as subtarefas $\mathrm{T} 1 \mathrm{com}$ três e quatro palavras.

A aplicação do PTCF é individual, sendo que para cada item de cada tarefa é realizado um treino anterior e após, são realizadas duas tentativas. Para resposta correta na primeira tentativa atribuise o valor 2 (dois); para cada resposta correta na segunda tentativa atribui-se o valor 1 (um); para as respostas erradas atribui-se o valor 0 (zero). Em cada tarefa o máximo de pontos que pode ser obtido é 10 (dez). Escores iguais ou superiores a 5 (cinco) indicam êxito em determinada tarefa, ou seja, aquisição consolidada de determinada habilidade em CF.

A avaliação da CPDF tem como objetivo fazer com que a criança ouça e julgue os desvios existentes em sua própria fala ${ }^{10}$. Para isso, são selecionadas de sua amostra de fala 10 palavras produzidas com desvio, as quais são editadas e gravadas isoladamente. Essas palavras são selecionadas aleatoriamente, mas considerando o inventário fonético/ fonológico de cada criança e a representatividade das alterações da fala da criança, na qual são identificados os processos fonológicos existentes.

Para que não haja dificuldade em identificar a palavra-alvo é indicada a seleção de figuras correspondentes às palavras selecionadas da amostra de fala para que sejam mostradas no momento da aplicação do teste. Um instrumento individual é montado, conforme o sistema fonológico (com base nos dados da coleta de fala) para cada sujeito.

Posteriormente, num intervalo de tempo de aproximadamente uma semana as palavras são apresentadas à criança de forma descontextualizada no intuito de dificultar que ela perceba que se tratam de palavras produzidas por ela mesma. É explicado à criança que ela escutará dez palavras faladas "por uma outra criança" e que deverá julgar se foram produzidas de forma adequada ou inadequada. Para cada uma das dez palavras selecionadas mostra-se a figura, aciona-se o gravador para que ela escute a produção oral da palavra e diga se a palavra foi, ou não foi produzida de maneira correta. Após o julgamento das dez palavras, elas devem ser apresentadas mais uma vez para que sejam julgadas novamente, ou seja, a criança deve ouvir duas vezes as dez palavras produzidas com desvio por ela própria.

As duas tentativas de julgamento de cada palavra são valoradas, sendo que os julgamentos corretos valem 1 (um) ponto e os incorretos valem 0 (zero). O máximo de pontos possíveis de serem alcançados é 20 (vinte) e, após a contagem dos pontos de cada criança é realizada uma média geral do grupo que está sendo avaliado. A criança não é avisada, em momento algum, de que as palavras foram produzidas por ela para evitar, segundo as autoras, que fatores emocionais interfiram no teste ${ }^{10}$.

Depois de realizadas as avaliações, as crianças foram divididas em grupos considerando a classificação quantitativa e qualitativa utilizadas neste estudo. Conforme a classificação quantitativa, as crianças foram divididas nos seguintes grupos: grupo com Desvio Médio (GDM) com seis sujeitos, grupo com Desvio Médio-Moderado (GDMM) com cinco sujeitos e grupo com Desvio ModeradoSevero (GDMS) com três sujeitos. De acordo com a classificação qualitativa, foram formados dois grupos: grupo com características Atrasadas (GCA) com nove sujeitos e grupo com características Iniciais $(\mathrm{GCl})$ com cinco sujeitos.

Este trabalho faz parte de um projeto maior intitulado "A estratégia de alongamento compensatório e sua relação com as habilidades em consciência fonológica", que foi aprovado pelo Comitê de Ética em Pesquisa do Centro de Ciências da Saúde da Universidade Federal de Santa Maria, com cadastro sob número 0103.0.243.000-07. Os pais e/ou 
responsáveis das crianças e as instituições (Serviço de Atendimento Fonoaudiológico e escola), foram devidamente esclarecidos sobre os objetivos e procedimentos da pesquisa, autorizando a participação das mesmas por meio do Termo de Consentimento Livre e Esclarecido e Consentimento Institucional, respectivamente.

Foi analisada a diferença no desempenho em tarefas de habilidades em CF e a CPDF apresentadas pelos sujeitos entre os grupos, considerando a classificação quantitativa e qualitativa separadamente. Os dados foram agrupados em tabelas de frequência considerando-se os grupos dos diferentes tipos de classificação dos DF, as diferentes tarefas em CF e a CPDF. Com essa tabulação, foram selecionados os dados pertinentes à pesquisa, os quais foram submetidos à análise estatística por meio do Teste estatístico não-paramétrico Kruskal-Wallis, considerando um nível de significância de 5\%.

\section{RESULTADOS}

Os resultados foram analisados considerando os dois tipos de classificação dos DF, qualitativa e quantitativa. Tanto para o $\mathrm{GCA}$ e o $\mathrm{GCl}$, quanto para o GDM, GDMM e o GDMS, foram analisados o desempenho nas habilidades em CF com a análise de cada subtarefa do teste utilizado e, na CPDF.

Considerando o GCA e o $\mathrm{GCl}$ pode-se observar diferenças estatisticamente significantes nas tarefas T2 - realismo nominal $(p=0,049)$; T6 inicial - detecção de sílaba $(p=0,049)$ e T6 medial - detecção de sílaba $(p=0,028)$ (Tabela 1).

Para o GDM, GDMM e o GDMS não houve diferença estatisticamente significante no que se refere às habilidades em CF (Tabela 2).

Quanto ao teste de CPDF não houve diferença estatisticamente significante entre o GCA e o GCI (Tabela 3). Já para o GDM, GDMM e o GDMS houve diferença estatística para essa variável (Tabela 4). Tal diferença foi influenciada, principalmente, pela diferença estatisticamente significante quando comparados o GDMM e o GDMS ( $p=0,0253)$.

\section{DISCUSSÃO}

Na Tabela 1 observou-se que houve diferença estatisticamente significante entre os grupos em três das 17 subtarefas aplicadas do PTCF quando os DF foram classificados de maneira qualitativa. De acordo com as médias, embora os dois grupos tenham obtido êxito nessas três subtarefas correspondentes às habilidades de realismo nominal e

Tabela 1 - Desempenho dos sujeitos nas habilidades em Consciência Fonológica de acordo com a classificação qualitativa dos desvios fonológicos *

\begin{tabular}{|c|c|c|c|c|c|c|c|}
\hline \multirow{2}{*}{ Sub-tarefa } & \multicolumn{3}{|c|}{ GCA } & \multicolumn{3}{|c|}{ GCl } & \multirow{2}{*}{ Valor de $p$} \\
\hline & $n$ & média & D.P. & $n$ & média & $D . P$. & \\
\hline T1 duas palavras & 9 & 6.8888889 & 3.0184617 & 5 & 7.6000000 & 2.8809721 & 0.5884 \\
\hline T1 três palavras & 2 & 4.0000000 & 4.2426407 & 4 & 6.0000000 & 2.8284271 & 0.3476 \\
\hline T1 quatro palavras & 2 & 2.0000000 & 2.8284271 & 4 & 4.5000000 & 1.2909944 & 0.2403 \\
\hline T2 & 9 & 7.5555556 & 2.1278576 & 5 & 5.0000000 & 1.8708287 & $0.0498^{*}$ \\
\hline T3 dissílabas & 9 & 8.1111111 & 2.4720662 & 5 & 5.8000000 & 4.2071368 & 0.2117 \\
\hline T3 trissílabas & 9 & 7.8888889 & 1.6914819 & 5 & 7.0000000 & 2.1213203 & 0.3409 \\
\hline T4 dissílabas & 9 & 9.5555556 & 1.3333333 & 5 & 10.0000000 & 0 & 0.4561 \\
\hline T4 trissílabas & 9 & 9.0000000 & 2.0000000 & 5 & 8.8000000 & 1.7888544 & 0.7556 \\
\hline T4 quadrissílabas & 9 & 8.2222222 & 2.7284509 & 5 & 8.8000000 & 1.7888544 & 0.8235 \\
\hline T5 dissílabas & 9 & 8.7777778 & 2.2236107 & 5 & 7.8000000 & 3.8987177 & 0.6977 \\
\hline T5 trissílabas & 9 & 9.2222222 & 1.0929064 & 5 & 9.0000000 & 1.7320508 & 1.0000 \\
\hline T5 quadrissílabas & 9 & 6.8888889 & 2.7588242 & 5 & 6.4000000 & 2.6076810 & 0.7352 \\
\hline T6 inicial & 9 & 8.5555556 & 1.6666667 & 5 & 5.8000000 & 2.8635642 & $0.0493^{\star}$ \\
\hline T6 final & 9 & 7.5555556 & 1.1303883 & 5 & 6.0000000 & 3.3166248 & 0.6312 \\
\hline T6 medial & 9 & 7.3333333 & 2.5495098 & 5 & 5.0000000 & 2.8284271 & $0.0284^{\star}$ \\
\hline T7 dissílaba & 7 & 4.8571429 & 1.3451854 & 1 & 0 & . & 0.1221 \\
\hline T9 inicial & 9 & 5.6666667 & 2.2360680 & 5 & 3.8000000 & 2.1679483 & 0.0759 \\
\hline T9 final & 7 & 7.5714286 & 1.7182494 & 1 & 8.0000000 & . & 0.8220 \\
\hline
\end{tabular}

GCA - grupo com características Atrasadas;

$\mathrm{GCl}$ - grupo com características Iniciais.

** Teste Kruskal-Wallis

* Valor de $p<0,05$

$\mathrm{n}$ : número de sujeitos

D.P.: desvio padrão 
Tabela 2 - Desempenho dos sujeitos nas habilidades em Consciência Fonológica de acordo com a classificação quantitativa dos desvios fonológicos **

\begin{tabular}{|c|c|c|c|c|c|c|c|c|c|c|}
\hline \multirow{2}{*}{ Sub-tarefa } & \multicolumn{3}{|c|}{ GDM } & \multicolumn{3}{|c|}{ GDMM } & \multicolumn{3}{|c|}{ GDMS } & \multirow{2}{*}{ Valor de $p$} \\
\hline & $n$ & Média & D.P. & $n$ & média & D.P. & $n$ & média & D.P. & \\
\hline T1 duas palavras & 6 & 5.8333333 & 3.0605010 & 5 & 8.0000000 & 3.0822070 & 3 & 8.3333333 & 1.5275252 & 0.2341 \\
\hline T1 três palavras & 1 & 1.0000000 & & 2 & 7.5000000 & 0.7071068 & 3 & 5.3333333 & 3.0550505 & 0.2519 \\
\hline T1 quatro palavras & 1 & 0 & . & 2 & 4.0000000 & 0 & 3 & 4.6666667 & 1.5275252 & 0.2936 \\
\hline T2 & 6 & 7.5000000 & 2.3452079 & 5 & 6.4000000 & 2.8809721 & 3 & 5.3333333 & 0.5773503 & 0.2771 \\
\hline T3 dissílabas & 6 & 7.8333333 & 2.7141604 & 5 & 7.6000000 & 3.0495901 & 3 & 5.6666667 & 5.1316014 & 0.7947 \\
\hline T3 trissílabas & 6 & 7.8333333 & 2.1369761 & 5 & 7.0000000 & 1.7320508 & 3 & 8.0000000 & 1.7320508 & 0.8620 \\
\hline T4 dissílabas & 6 & 9.3333333 & 1.6329932 & 5 & 10.0000000 & 0 & 3 & 10.0000000 & 0 & 0.5134 \\
\hline T4 trissílabas & 6 & 8.6666667 & 2.4221203 & 5 & 9.4000000 & 0.8944272 & 3 & 8.6666667 & 2.3094011 & 0.9874 \\
\hline T4 quadrissílabas & 6 & 7.6666667 & 3.2041640 & 5 & 9.2000000 & 1.0954451 & 3 & 8.6666667 & 2.3094011 & 0.7607 \\
\hline T5 dissílabas & 6 & 8.8333333 & 2.4013885 & 5 & 7.4000000 & 3.9749214 & 3 & 9.3333333 & 1.1547005 & 0.8607 \\
\hline T5 trissílabas & 6 & 8.8333333 & 1.1690452 & 5 & 9.2000000 & 1.7888544 & 3 & 9.6666667 & 0.5773503 & 0.4329 \\
\hline T5 quadrissílabas & 6 & 5.6666667 & 2.5033311 & 5 & 7.6000000 & 3.2863353 & 3 & 7.3333333 & 1.1547005 & 0.2841 \\
\hline T6 inicial & 6 & 8.5000000 & 2.0736441 & 5 & 8.2000000 & 1.3038405 & 3 & 4.6666667 & 3.0550505 & 0.0974 \\
\hline T6 final & 6 & 7.5000000 & 1.3784049 & 5 & 7.8000000 & 0.8366600 & 3 & 4.6666667 & 3.7859389 & 0.4538 \\
\hline T6 medial & 6 & 6.8333333 & 3.0605010 & 5 & 7.4000000 & 1.3416408 & 3 & 4.3333333 & 3.7859389 & 0.2748 \\
\hline T7 dissílaba & 5 & 4.4000000 & 1.1401754 & 3 & 4.0000000 & 3.6055513 & 0 & ${ }^{\circ}$ & . & 0.7628 \\
\hline T9 inicial & 6 & 6.1666667 & 2.1369761 & 5 & 4.6000000 & 1.8165902 & 3 & 3.3333333 & 2.8867513 & 0.1227 \\
\hline T9 final & 5 & 8.2000000 & 1.6431677 & 3 & 6.6666667 & 1.1547005 & 0 & & . & 0.1665 \\
\hline
\end{tabular}

GDM - grupo com Desvio-Médio

GDMM - grupo com Desvio Médio-Moderado

GDMS - grupo com Desvio Moderado-Severo

** Teste Kruskal-Wallis

* Valor de $p<0,05$

n: número de sujeitos

D.P.: desvio padrão

Tabela 3 - Comparação da consciência do próprio desvio de fala (CPDF) entre os grupos conforme a classificação qualitativa dos desvios fonológicos *

\begin{tabular}{c|ccc|ccc|c}
\hline & \multicolumn{4}{|c|}{ GCA } & \multicolumn{4}{|c|}{ GCI } & Valor de $\mathrm{p}$ \\
\hline \multirow{2}{*}{ CPDF } & $n$ & Média & D.P. & $n$ & média & D.P. & \multirow{2}{*}{0.3474} \\
\cline { 2 - 7 } & 9 & 7.2222222 & 6.6101773 & 5 & 3.4000000 & 3.6469165 & \\
\hline
\end{tabular}

CPDF - Consciência do Próprio Desvio de Fala

GCA - grupo com características Atrasadas;

$\mathrm{GCl}$ - grupo com características Iniciais.

** Teste Kruskal-Wallis

* Valor de $p<0,05$

n: número de sujeitos

D.P.: desvio padrão

Tabela 4 - Comparação da consciência do próprio desvio de fala (CPDF) entre os grupos conforme a classificação quantitativa dos desvios fonológicos **

\begin{tabular}{|c|c|c|c|c|c|c|c|c|c|c|}
\hline \multirow{3}{*}{ CPDF } & \multicolumn{3}{|c|}{ GDM } & \multicolumn{3}{|c|}{ GDMM } & \multicolumn{3}{|c|}{ GDMS } & Valor de $p$ \\
\hline & $n$ & média & D.P. & $n$ & média & D.P. & $n$ & média & D.P. & \multirow{2}{*}{$0.0376^{*}$} \\
\hline & 6 & 3.8333333 & 4.1673333 & 5 & 11.2000000 & 5.5407581 & 3 & 1.0000000 & 1.0000000 & \\
\hline
\end{tabular}

CPDF - Consciência do Próprio Desvio de Fala

GDM - grupo com Desvio-Médio

GDMM - grupo com Desvio Médio-Moderado

GDMS - grupo com Desvio Moderado-Severo

** Teste Kruskal-Wallis

* Valor de $p<0,05$

n: número de sujeitos

D.P.: desvio padrão 
detecção de sílabas, o GCA obteve melhores resultados em todas elas.

Quando classificados de forma quantitativa, os DF não apresentaram diferenças estatisticamente significantes em nenhuma das tarefas do PTCF (Tabela 2). No que se refere às tarefas de detecção de sílaba, o GDMS não obteve êxito, diferente da classificação qualitativa em que o GCA e o GCl apresentaram êxito nesta habilidade.

Tais achados concordam parcialmente com estudo que apresentou como resultado, êxito na tarefa de realismo nominal por crianças com desenvolvimento fonológico normal na faixa etária de quatro a oito anos de idade, sendo que houve diferença estatisticamente significante aos sete e oito anos para essa tarefa ${ }^{12,13}$. Considerando que as crianças do presente estudo inserem-se na faixa etária de cinco a seis anos de idade supõe-se que os resultados encontrados para a tarefa T2 entre o GCA e o $\mathrm{GCl}$ possam ter sido influenciados pela classificação do DF, uma vez que o $\mathrm{GCl}$ apresenta características específicas do inicio da aquisição da linguagem. A tarefa T2 do PTCF corresponde ao realismo nominal que é a habilidade da criança em distanciar o referente (objeto) do seu signo semiológico (palavra).

Os resultados obtidos para a tarefa T6 na classificação qualitativa corroboram os achados em que essa subtarefa foi realizada com êxito por crianças com desenvolvimento fonológico normal nas faixas etárias de cinco, seis, sete e oito anos de idade ${ }^{12,13}$. Para o GCA houve facilidade na seguinte sequência: posição inicial, seguida da posição final e, por último, a posição medial para a tarefa T6, de acordo com os estudos citados. Para esta tarefa, os melhores resultados com diferença estatisticamente significante, de acordo com a média, foram para o GCA supondo-se mais uma vez que a classificação do DF possa ter influenciado nos resultados. Quando considerada a classificação quantitativa os resultados não concordam com os estudos referidos já que para o GDMS não foi obtido êxito na tarefa em questão. A tarefa T6 do PTCF corresponde à detecção de sílabas a qual avalia a habilidade da criança em perceber e localizar sílabas dentro de palavras orais.

Os achados neste estudo para a subtarefa T6 inicial divergem dos resultados encontrados em um estudo sobre o desempenho em habilidades de rima e aliteração realizado com quarenta sujeitos, com idades entre quatro e oito anos, classificados em diferentes hipóteses de escrita. Esse estudo apontou uma maior facilidade para a tarefa de "identificação de sílaba inicial" para todos os sujeitos ${ }^{11}$.

Analisando de forma geral, o GCA e o $\mathrm{GCl}$ apresentaram, em ordem decrescente, melhores desempenhos nas subtarefas que envolvem consciência silábica, rima e aliteração. Estes resultados vão ao encontro de um estudo que investigou as habilidades em CF em crianças com DF em que também se observou melhores desempenhos nos subtestes referidos. Esses achados revelam que a emergência das habilidades em CF em crianças com DF ocorre, de maneira geral, de modo semeIhante ao evidenciado normalmente pelas crianças e com alguma previsibilidade ${ }^{19}$.

Para o GDM e o GDMM os melhores resultados também foram obtidos nas subtarefas correspondentes a consciência silábica, rima e aliteração em ordem decrescente. O GDMS, por outro lado, não obteve êxito nas subtarefas de detecção de sílabas e de fonemas. Tal resultado pode ter sido influenciado pela classificação dos DF, pois dos três grupos o GDMS é o que apresenta uma maior gravidade dos desvios (Tabela 2).

De acordo com as médias obtidas para a classificação qualitativa, notou-se que ambos os grupos não obtiveram êxito em três das subtarefas, no entanto, de maneira geral, o GCA obteve melhores desempenhos na maioria das subtarefas em relação ao GCl (Tabela1). Para a classificação quantitativa o grupo com gravidade mais severa - GDMS - foi o que não obteve êxito em um maior número de subtarefas do PTCF quando comparados com os outros grupos da sua classificação. Tal grupo não obteve êxito nas subtarefas de segmentação de frases (T1 com quatro palavras), detecção de sílabas (T6 inicial, final e medial) e detecção de fonemas (T9 inicial) (Tabela 2). Esses achados sugerem uma possível relação entre a gravidade do DF e o desempenho nas habilidades em CF.

No intuito de analisar a relação entre os tipos de erros de fala produzidos por crianças com transtornos da fala e habilidades em CF foi demonstrado que pode haver uma relação entre as variáveis referidas. Nesse estudo 58 crianças foram avaliadas em dois momentos quanto aos erros produzidos na fala e a CF em idades pré-escolar e escolar. As crianças que falharam no teste de CF em idade pré-escolar produziram, de forma significativa, mais erros típicos de estrutura silábica (como redução de encontro consonantal e apagamento de consoante final) quando comparados às crianças que passaram. As crianças em idade escolar que falharam no teste de CF produziram, de forma significativa, mais erros atípicos de segmentos (como semivocalização de fricativa e posteriorização de fricativa alveolar) em relação às crianças que passaram. Os resultados obtidos nesse estudo apontam uma tendência para uma relação entre as características dos erros de fala e o risco da ocorrência de problemas nas habilidades em CF ${ }^{20}$. 
Em um estudo realizado com crianças de préescola apresentando atraso de linguagem e/ou DF e crianças com desenvolvimento normal constatouse que as crianças com dificuldade de fala apresentaram déficits nas habilidades em CF. Os autores acreditam que essas alterações podem ser minimizadas com um programa de intervenção para essas habilidades ${ }^{15}$.

Além do DF, o desenvolvimento da CF pode ser influenciado por outros fatores, dentre eles a percepção da fala. A percepção da fala e a CF incluem aspectos diferentes de processamento fonológico sendo que ambas as tarefas envolvem acesso acústico-articulatório e representações fonológicas dos itens lexicais. No entanto, a percepção da fala depende de representações de detalhes acústico-articulatórios das palavras, enquanto que a CF requer representações das mesmas no léxico. Com isso, crianças com DF podem apresentar um grande risco para um desenvolvimento lento das habilidades em CF ${ }^{21}$.

Ressalta-se ainda que a CF se interrelaciona com a habilidade de memória de trabalho que por sua vez tem relação com a gravidade do $\mathrm{DF}^{22}$. Em um grupo de crianças com DF de diferentes gravidades e idade média de seis anos foi observado que as crianças que repetiram palavras sem significado com um menor número de sílabas, apresentaram a fala mais ininteligível. Esses resultados ratificam a hipótese de que há associação entre a memória fonológica e a gravidade do DF ${ }^{23}$.

Conforme os resultados apresentados na Tabela 3 , observou-se que houve diferença estatisticamente significante somente entre o GDM, o GDMM e o GDMS em relação à CPDF. A média para o GDMM é maior em relação às médias do GDM e do GDMS o que sugere uma melhor CPDF por esses sujeitos. Para o GCA e o GCI não houve diferença estatística, porém, de acordo com as médias o GCA evidencia um melhor resultado no que se refere à CPDF.

As crianças com DF podem ter consciência do sistema fonológico considerado normal, pois demonstram capacidade de raciocinar sobre os sons do português respondendo a testes de CF. Sabe-se que o desenvolvimento da CF tem mútua relação com a aprendizagem da leitura e escrita, por isso, a CPDF pode servir como um facilitador para a não repercussão dos desvios orais na escrita, interferindo no desempenho de crianças com DF 10,24,25.

Os resultados obtidos nesse estudo demonstram que as crianças com DF possuem capacidade de refletir sobre os sons de sua língua e podem ter CPDF a qual parece sofrer influência da gravidade do DF.

Sugere-se que novas pesquisas, com um maior número de sujeitos, sejam realizadas no intuito de investigar as relações entre as habilidades em CF, a CPDF e diferentes gravidades do DF.

\section{CONCLUSÃO}

Os resultados obtidos neste estudo permitem inferir que há uma possível relação entre o desempenho nas habilidades em CF e a gravidade dos DF. Nos dois tipos de classificações dos DF utilizados - qualitativa e quantitativa - houve diferença no desempenho de algumas habilidades em CF entre os grupos. Para a classificação qualitativa houve diferenças significativas em algumas subtarefas. Além disso, os grupos com maior gravidade do DF foram os que apresentaram, de maneira geral, piores resultados nas habilidades pesquisadas em CF.

Com relação à CPDF pode-se concluir com os resultados apresentados e com base em outros estudos que as crianças com DF são capazes de refletir sobre os sons de sua língua, pois demonstram capacidade de refletir sobre os sons da fonologia do português, respondendo a testes de CF. Essas crianças podem ter CPDF, sendo capazes de reconhecer tais desvios quando os escutam. Contudo, o reconhecimento de um desvio de fala pela criança não garante que a mesma consiga corrigi-lo em sua própria fala.

Observou-se neste estudo que as crianças com graus menos severos de desvios apresentam maior consciência dos desvios de fala. De acordo com os resultados obtidos para ambas as classificações, pode-se observar uma provável influência da gravidade dos DF na CPDF. 


\begin{abstract}
Purpose: to examine the relation between abilities in phonological awareness and awareness of the own speech impairment with different severity levels of phonological disorders. Methods: 14 children took part in this study, aged between 5:0 and 6:11, showing phonological disorder diagnosis. Phonological disorders were classified in two perspectives: quantitative and qualitative. According to the quantitative approach, the groups were classified in Medium, Medium-Moderate and Moderate-Severe deviation. According to the qualitative approach there were the group with Delayed characteristics and the group with Initial characteristics. In order to collect the data, the Test of Phonological Awareness and the assessment of the awareness regarding their own speech impairment were adopted. Data were analyzed based on Kruskal-Wallis statistic test. Results: statistically significant differences were observed in the tasks of nominal realism and syllable detection among the groups classified according to the qualitative approach. For the groups classified according to the quantitative approach, there was not statistically significant difference in the Test of Phonological Awareness. However, considering the assessment, the awareness of their own speech impairment, just these last groups presented statistically significant differences among themselves. Conclusion: a possible relation was identified between the performance in the phonological awareness tasks and the different severity levels of phonological disorder, mainly in a qualitative approach. The awareness of their own speech impairment also seems to be influenced by the different severity levels of phonological disorder, mainly in a quantitative approach.
\end{abstract}

KEYWORDS: Speech; Speech Disorders; Child; Language

\section{REFERÊNCIAS}

1. Lamprecht RR. Antes de mais nada. In: Lamprecht $\mathrm{RR}$, organizador. Aquisição fonológica do Português: perfil de desenvolvimento e subsídios para a terapia. Porto Alegre: Artmed; 2004. p. 17-32.

2. Vieira MG, Mota HB, Keske-Soares M. Relação entre idade, grau de severidade do desvio fonológico e consciência fonológica. Rev Soc Bras Fonoaudiol. 2004; 9(3):144-50.

3. Keske-Soares M, Blanco APF, Mota HB. O desvio fonológico caracterizado por índices de substituição e omissão. Rev Soc Bras Fonoaudiol. 2004; 9(1): 10-8.

4. Yavas M, Hernandorena CLM, Lamprecht RR. Avaliação fonológica da criança. Porto Alegre: Artes Médicas; 1991.

5. Wertzner HF, Amaro L, Galea DES. Phonological performance measured by speech severity indices compared with correlated factors. São Paulo Med J. 2007; 125(6):309-14.

6. Shriberg LD, Kwiatkowski J. Phonological disorders I: a diagnostic classification system. J Speech Hear Disord. 1982; 47(3):226-41.

7. Shriberg LD, Kwiatkowski J. Phonological disorders III: a procedure for assessing severity of involvement. J Speech Hear Disord. 1982; 47(3):256-70.

8. Keske-Soares M. Terapia fonoaudiológica fundamentada na hierarquia implicacional dos traços distintivos aplicada em crianças com desvios fonológicos. [tese]. Porto Alegre (RS): Pontifícia Universidade Católica do Rio Grande do Sul; 2001.

9. Magnusson E. Consciência metalinguística em crianças com desvios fonológicos. In: Yavas M, organizador. Desvios fonológicos em crianças: teoria, pesquisa e tratamento. Porto Alegre: Mercado Aberto; 1990. p.109-48.

10. Menezes G, Lamprecht RR. A consciência fonológica na relação fala-escrita em crianças com desvios fonológicos evolutivos (DFE). Letras de Hoje. 2001; 36(3):743-9.

11. Menezes GC. Consciência fonológica: rimas e aliterações no português brasileiro. Letras de Hoje. 2003; 38(2):155-70.

12. Cielo CA. Habilidades em consciência fonológica em crianças de 4 a 8 anos de idade. Pró-Fono. 2002; 14(3):301-12.

13. Cielo CA. Avaliação de habilidades em consciência fonológica. J Bras Fonoaudiol. 2003; 4(16):163-74.

14. Maluf MR, Barrera SD. Consciência metalinguística e alfabetização: um estudo com crianças da primeira série do ensino fundamental. Psicol Reflex Crít. 2003; 16(3):491-502.

15. Laing SP, Espeland W. Low intensity phonological awareness training in a preschool classroom for children with communication impairments. J Commun Disord. 2005; 38(1):65-82. 
16. Sutherland D, Gillon GT. Development of phonological representations and phonological awareness in children with speech impairment. Int $\mathrm{J}$ Lang Commun Disord. 2007; 42(2):229-50.

17. Zorzi JL, Hage SRV. PROC - Protocolo de observação comportamental. São José dos Campos: Pulso; 2004. p.93.

18. Ferreiro E, Teberosky AA. Psicogênese da língua escrita. Porto Alegre: Artes Médicas; 1991. p.284.

19. Morales MV, Mota HB, Keske-Soares M. Consciência fonológica: desempenho de crianças com e sem desvios fonológicos evolutivos. PróFono. 2002; 14(2):153-64.

20. Rvachew S, Chiang PY, Evans N. Characteristics of speech errors produced by children with and without delayed phonological awareness skills. Lang Speech Hear Serv Schools. 2007; 38(1):60-71.

21. Rvachew S, Grawburg M. Correlates of phonological awareness in preschoolers with speech sound disorders. J Speech Lang Hear Res. 2006; 49(1):74-87.
22. Gindri G, Keske-Soares M, Mota HB. Memória de trabalho, consciência fonológica e hipótese de escrita. Pró-Fono. 2007; 19(3):313-22.

23. Linassi LZ, Keske-Soares M, Mota H. Habilidades de memória de trabalho e o grau de severidade do desvio fonológico. Pró-Fono. 2005; 17(3):383-92.

24. França PM, Wolff CL, Moojem S, Rotta NT. A aquisição da linguagem oral: relação e risco para a linguagem escrita. Arq Neuro-Psiquiatr. 2004; 62(2B):469-72.

25. Mota HB, Melo Filha MGC, Lasch SS. A consciência fonológica e o desempenho na escrita sob ditado de crianças com desvio fonológico após realização de terapia fonoaudiológica. Rev CEFAC. 2007; 9(4):477-82. http://dx.doi.org/10.1590/ S1516-18462007000400007

DOI: 10.1590/S1516-18462009005000041

RECEBIDO EM: 04/07/2008

ACEITO EM: 05/04/2009

Endereço para correspondência:

Roberta Freitas Dias

Rua Antero Corrêa de Barros, 244

Santa Maria - RS

CEP: 97010-120

E-mail: r_fdias@hotmail.com 